\title{
EDITORIAL
}

\section{HIV in children: take a moment to make a difference!}

\author{
Savvas Andronikou
}

Received: 30 March 2009/Accepted: 5 April 2009/Published online: 5 May 2009

(C) Springer-Verlag 2009

You may not know it, but there are 5.5 million HIV-infected people in Africa. More important for paediatric radiologists to know is that there are nearly 2 million HIV-infected children in sub-Saharan Africa. In my home country, South Africa, there are 360,000 HIV-infected children. Now you understand why we have dedicated seven articles in this minisymposium to HIV in children. You may not believe that this affects you as a European or North American radiologist, but if the resurgence of tuberculosis is anything to go by, HIV should be a major concern to you too. The mass migration from Africa, increasing urban poverty and worsening social conditions in parts of cities of many developed nations make Africa at your doorstep.

The majority of adults with HIV infection are of childbearing age, and of these, three-quarters are women. This, in turn, translates to both a medical and a social disaster for children. Not only are the bread-winners ill and not able to care for their children, but mothers transmit the disease during pregnancy, delivery and breast feeding to their children. Additionally, parents who are not treated and die leave orphans behind (many of whom are infected with the virus). There are currently over 11 million AIDS orphans!

Imaging in HIV infection and AIDS requires skills ranging from interpretation of plain radiographs to MRI scanning of the central nervous system. Children with HIV infection are susceptible to both common and unusual infections, sometimes simultaneously. The radiologist also needs to distinguish changes resulting from HIV infection

S. Andronikou $(\square)$

Department of Radiology, Tygerberg Hospital,

203 Mullerhof, 15 Belle Ombre Road, Tamboerskloof,

8001 Cape Town, South Africa

e-mail: docsav@mweb.co.za itself, such as brain shrinkage or cardiomegaly. Furthermore, neurological damage, chronic illness and superinfection compound each other with a variety of complications, such as aspiration and cardiac failure. Neoplasms, such as lymphoma, present radiologically in similar ways to some infections such as TB, when lymphadenopathy can no longer be used as a distinguishing imaging feature. The patient's condition also affects the diseases that they are most susceptible to, and depending on CD4 levels the composition of a radiological differential diagnosis changes. Moreover, the institution of highly active antiretroviral therapy further complicates image interpretation, especially when the body is able to mount an immune response, resulting in the phenomenon of immune reconstitution inflammatory syndrome (IRIS).

Without information from the clinician, the radiologist can become lost in identifying multiple radiological signs without any coherent conclusion and without being able to assist the clinician to manage the patient. Children may be on prophylaxis for pneumocystis, making that an unlikely diagnosis. There may be newonset pyrexia and the suggestion of fungal disease. Coexistent skin lesions may allow the rare diagnosis of Kaposi sarcoma in the lung to be made on radiographic findings. The clinician may depend on radiological identification of lymphadenopathy to start anti-TB treatment. The CD4 count may make some infections or neoplasms less likely and others more likely.

Imaging is also useful for follow-up. Disease progression, resolution of acute infections, response to treatment and success of interventions for some complications can be determined this way.

Because HIV predominantly affects children in the developing world, imaging research should be geared towards low-cost solutions. However, high-end imaging in 
specialized centres, including multidetector $\mathrm{CT}$ reconstructions of TB lymphadenopathy for determining the most reliable plain radiographic signs, diffusion tensor imaging for determining whether CT-visible atrophy relates to white-matter destruction or reversible volume loss, and MRI scanning in complications such as TB meningitis for CSF flow dynamics are underway. The important thing with HIV research is for the results to be translated into practical solutions that enhance available resources and assist children in need.
The articles that we have collected will give you some insight into the magnitude of the HIV pandemic, the critical importance of relations with referring clinicians in this disease, and the most important imaging presentations. I hope that all paediatric radiologists will see it as a responsibility to be familiar with this disease and that they will somehow contribute research time, teaching time or working time to assist those children suffering in the developing world where radiologists are few and far between. 